\title{
The influence of contact area between implant and its adjacent teeth on finite element analysis
}

\author{
Wenjie Dai ${ }^{1}, \mathrm{Xi} \mathrm{Lu}^{2}$ \\ School of Mechanical Engineering, The University of Southeast, Nanjing, China \\ ${ }^{2}$ Corresponding author \\ E-mail: ${ }^{1}$ daiwenjie0603@163.com, ${ }^{2}$ seu_luxi@163.com \\ Received 9 January 2019; accepted 22 January 2019 \\ DOI https://doi.org/10.21595/vp.2019.20534
}

Check for updates

Copyright (C) 2019 Wenjie Dai, et al. This is an open access article distributed under the Creative Commons Attribution License, which permits unrestricted use, distribution, and reproduction in any medium, provided the original work is properly cited.

\begin{abstract}
The aim of this work is to investigate the influence of contact area between the first molar implant and its adjacent teeth on finite element analysis. Five types of finite element contact models containing implant and its adjacent teeth were established: non-contact model $(\mathrm{C} 0)$, contact models with different contact area $(\mathrm{C} 1, \mathrm{C} 2, \mathrm{C} 3, \mathrm{C} 4)$. An occlusal force was applied to finite element models to calculate the stress of implant and bone interface on mandible. Compared with the group $\mathrm{C} 0$, the stress of alveolar bone surrounding the implant in other groups decreased $88.08 \%$ at most, but the stress of cancellous bone surrounding the natural teeth increased $59.42 \%$. In summary, a proper small contact area can not only reduce the stress concentration on the implant region, but also can avoid an excessive stress in the periodontal ligament and alveolar bone around the natural teeth. The finite element results revealed that it is effectively to distribute the load implant suffered, especially to reduce the stress generated by the lateral load when the first molar implant has a small contact with its adjacent teeth.
\end{abstract}

Keywords: implant, contact area, adjacent teeth, finite element.

\section{Introduction}

Because of the protection of periodontal ligament, the load applied on teeth can be effectively absorbed [1], then the stress on alveolar bone is evenly distributed [2]. The surface of implant combines with the alveolar bone to form a bone interface after the implant is implanted into the correct position on mandible, this process achieves the aim of force transmission and repair the ability of chewing. Therefore, the key to a long-term successful implanting is the reliability and stability of the bone interface combination. Also, the stress of the bone interface plays an important role in evaluation index. Without the protection of periodontal ligament, stress concentration on the bone interface can be found easily, which generated by physiological load especially the lateral force. Paper about optimal design of implant diameter, length, material have been reported [3-5] to increase the success rate of implanting. However, there is rarely paper about the contact area between implant and its adjacent teeth. The condition of contact area between two teeth is usually ignored or simplified $[6,7]$ when a finite element of mandible is analyzed. In order to explore the effect of the contact area between implant and its adjacent teeth on the stress difference, five types of different contact areas with no contact as the control group were designed and simulated the situation of oral model to provide technical support for clinical application.

\section{Materials and methods}

\subsection{Model}

A 23-year-old female mandible CT image slices were used to reconstruct the model of mandible and dentition with the medical reverse modeling software Mimics. The periodontal ligament that could not be extracted from CT was obtained from the Shelling and Boolean operations according to the thickness in the paper [8]. The implant was scanned and the three-dimension of implant was obtained by reverse modeling in Geomagic. 
The statistical results of the area of contact surface between the first molar and the second premolar is $6.05 \pm 2.31 \mathrm{~mm}^{2}$ [9]. The implant crown of each group is amplified differently with the middle point of the upper surface of the crown as the center, the condition of contact area of the implant crown and its adjacent teeth is shown in Fig. 1. The group $\mathrm{C} 0$ shows the gap between the implant crown and its adjacent teeth; the group $\mathrm{C} 1$ shows the point contact between implant crown and its adjacent teeth, the area implant contact with the second premolar is $0.1275 \mathrm{~mm}^{2}$ and contact with the second molar and $0.2851 \mathrm{~mm}^{2}$. The area implant contact with the second premolar is $1.772 \mathrm{~mm}^{2}$ of group C2, $5.295 \mathrm{~mm}^{2}$ of group C3, $8.62 \mathrm{~mm}^{2}$ of group C4, and contact with the second molar is $2.095 \mathrm{~mm}^{2}$ of group C2, $6.58 \mathrm{~mm}^{2}$ of group C3, $11.284 \mathrm{~mm}^{2}$ of group C4, respectively. The model of the mandible including the implant was meshed using a 10 -node tetrahedral unit (C3D10M).

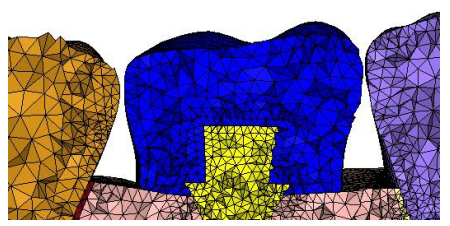

a) Original model

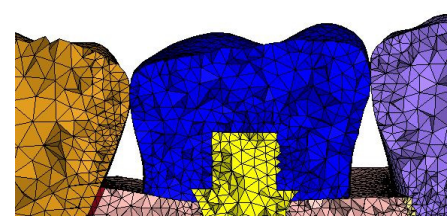

b) Enlarged by 1.04 times

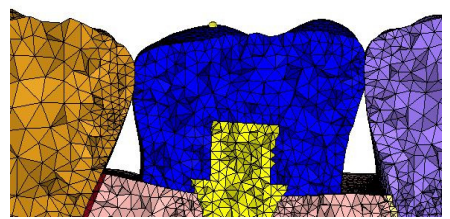

c) Enlarged by 1.06 times

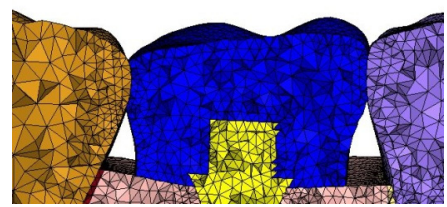

d) a) Enlarged by 1.1 times

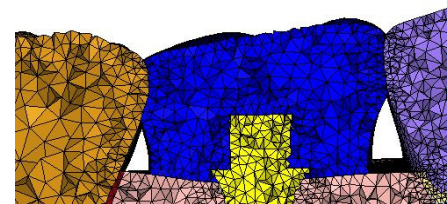

e) Enlarged by 1.15 times

Fig. 1. Contact conditions of implant and its adjacent teeth

\subsection{Material properties}

The materials of periodontal ligament, implant, the cortical bone, the cancellous bone, crown and tooth enamel were considered continuous, homogeneous, isotropic linear elastic materials $[8,10]$. Material properties are present in Table 1.

Table 1. Material properties

\begin{tabular}{|c|c|c|}
\hline Material & Modulus of elasticity (MPa) & Poisson's ratio \\
\hline Cortical bone & 13700 & 0.3 \\
\hline Cancellous bone & 2000 & 0.3 \\
\hline Tooth enamel & 18600 & 0.31 \\
\hline Periodontal ligament & 40 & 0.45 \\
\hline Implant & 110000 & 0.35 \\
\hline Crown & 200000 & 0.31 \\
\hline
\end{tabular}

\subsection{Boundary conditions}

The connections in the model among the periodontal ligament, the natural tooth, the cortical bone, and the cancellous bone are $100 \%$ connected, the crown and the abutment are connected by a common node, and the rest are applied tie connection. The coefficient of friction between natural teeth and the crown of implant in group $\mathrm{C} 1, \mathrm{C} 2, \mathrm{C} 3$ and $\mathrm{C} 4$ was 0.2 [11]. A local coordinate system is established for the second premolar, the first molar implant, and the second molar, respectively, of which the $Z$-axis is parallel to the longitudinal axis of the tooth and the $X$-axis is perpendicular to the dental surface of buccal side.

A longitudinal axial load of $120 \mathrm{~N}$ was applied to the cusp of the second premolar and $150 \mathrm{~N}$ was applied to the cusp of the second molar. A device for measuring the bite force is designed 
based on Testa's test [12], as shown in Fig. 2(a), which verified the finite element model of Fig. 2(b), the error of force of the experiment and the finite element model is $28.57 \%$. The bite force of first molar was calculated by the finite element model, which was applied in the cusp about $174 \mathrm{~N}$ in the vertical direction and $54 \mathrm{~N}$ in the horizontal direction. Six degrees of freedom are limited to the nodes of the $2 / 5$ part of the outer surface of the cortical bone.

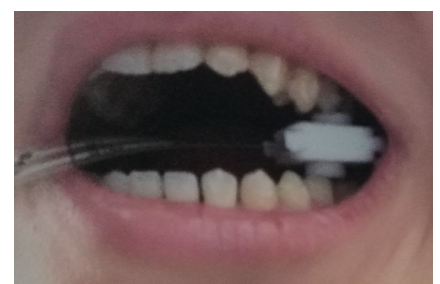

a) Experiment

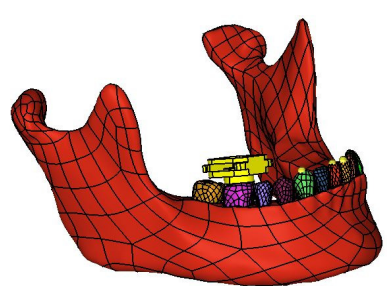

b) Finite element model

Fig. 2. Experimental verification of the finite element model

\section{Results}

\subsection{Stress on dental implants and the alveolar bone around implant}

The stress of the alveolar bone was circular distribution around the implant except for the cancellous bone of the $\mathrm{C} 0$ group. Stress concentration appeared on the lingual side of bone interface on the cancellous bone in $\mathrm{C} 0$ group. The values of stress on the cortical and cancellous bone surrounding the implant were illustrated in Fig. 3. Compared with the $\mathrm{C} 0$ group of $37.67 \mathrm{MPa}$ in cortical bone, the values of stress of $\mathrm{C} 4$ group is $14.43 \mathrm{MPa}$ and decreased $61.69 \%$. The stress of the cancellous bone in the non-contact model (C0) is $44.25 \mathrm{MPa}$, while those in the $\mathrm{C} 4$ group is $5.276 \mathrm{MPa}$ and decreased $88.08 \%$. It appears that within a certain range, the contact area between the first molar implant and its adjacent teeth is larger, the stress of the alveolar bone around the implant region is lower.

The values of stress on the implant were illustrated in Fig. 4. The stress mainly concentrated on the crown and the threaded joint of the implant, and the maximum value appears on the neck of the abutment for the model of five groups.

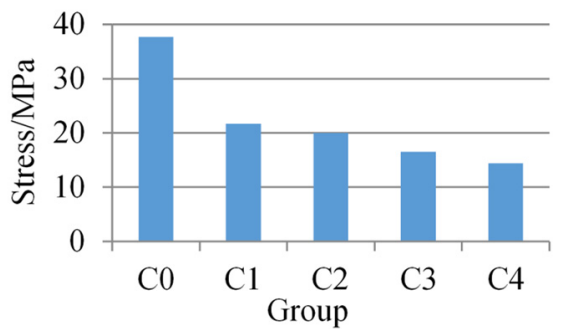

a) Cortical bone

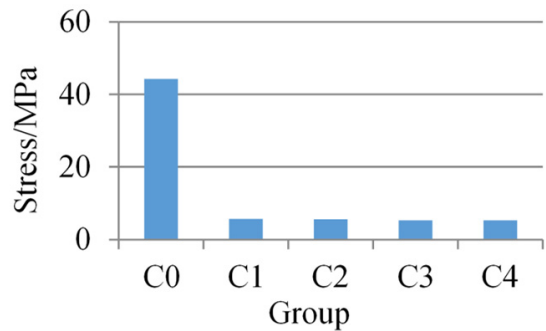

b) Cancellous bone

Fig. 3. Stress of cortical and cancellous bone surrounding the implant

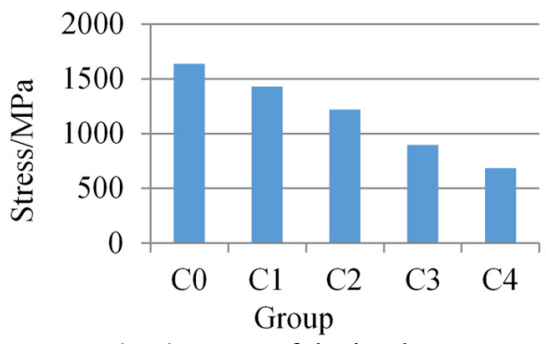

Fig. 4. Stress of the implant 
The stress of the neck of the abutment in the non-contact model (C0) is $1636 \mathrm{MPa}$, while those in the group $\mathrm{C} 4$ is $685.6 \mathrm{MPa}$ and decreased $58.09 \%$. It appears that within a certain range, the contact area between the first molar implant and its adjacent teeth is larger, the stress of the implant is lower.

\subsection{Stress on periodontal ligament and the alveolar bone around natural teeth adjacent to the implant}

The values of stress on the alveolar bone around natural teeth adjacent to the implant were illustrated in Fig. 5. The stress of the alveolar bone was circular distribution.

Fig. 5(a) demonstrates that the stress on the cortical bone around second premolar in the $\mathrm{C} 0$ is 23.8 $\mathrm{MPa}$, while those in the $\mathrm{C} 1$ group is $14.71 \mathrm{MPa}$ and decreased $38.19 \%$. Additionally, the values of stress of $\mathrm{C} 4$ group is $17.08 \mathrm{MPa}$ in cortical bone and increased $16.11 \%$, compared with the $\mathrm{C} 1$ group. The same situation happened on the cortical bone around second molar. The maximum stress of the cancellous bone appears at the bottom of the root, Fig. 5(b) shows that the stress on the cancellous bone around second premolar in the $\mathrm{C} 0$ is $5.505 \mathrm{MPa}$, while those in the C4 group is $8.08 \mathrm{MPa}$ and increased $46.78 \%$. Additionally, the values of stress of C4 increased $5.69 \%$, compared with the stress of $\mathrm{C} 1$ group $(7.645 \mathrm{MPa})$. The same situation happened on the cancellous bone around second molar. It appears that contacting with adjacent teeth can decrease the stress of cortical bone, which can increase the stress of cortical bone simultaneously. Moreover, within a certain range, the contact area between the first molar implant and its adjacent teeth is larger, the stress of the alveolar bone around natural teeth adjacent to the implant is higher.

The values of stress on the periodontal ligament of the natural teeth adjacent to the implant were illustrated in Fig. 6. The stress mainly appears at the root of periodontal ligament. The stress on the periodontal ligament of second premolar and second molar of group $\mathrm{C} 1$ is $3.705 \mathrm{MPa}$ and $2.056 \mathrm{MPa}$, while those in the C4 group is $5.557 \mathrm{MPa}$ and $2.864 \mathrm{MPa}$, and increased $49.99 \%$ and $39.30 \%$ respectively.

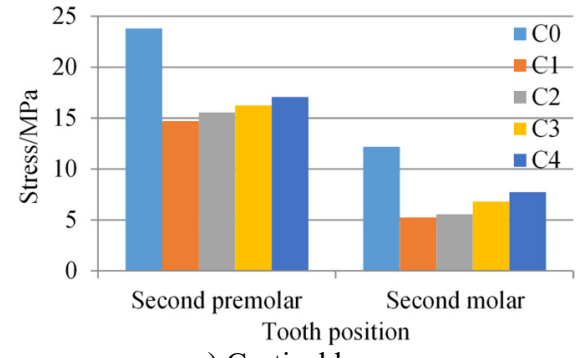

a) Cortical bone

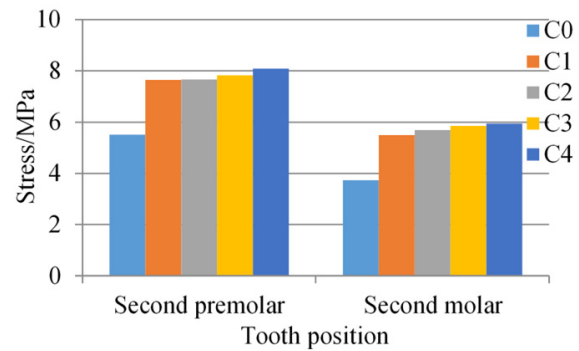

b) Cancellous bone

Fig. 5. Stress of the alveolar bone around natural teeth adjacent to the implant

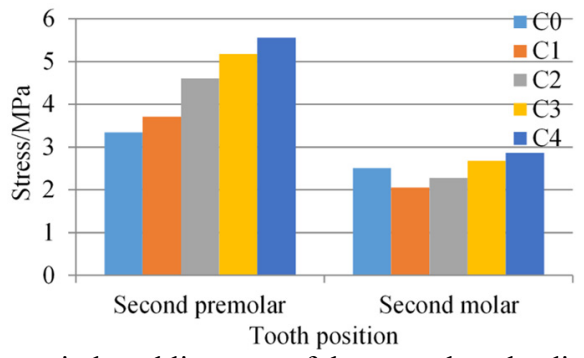

Fig. 6. Stress of the periodontal ligament of the natural teeth adjacent to the implant

It appears that within a certain range, the contact area between the first molar implant and its adjacent teeth is larger, the stress of the periodontal ligament of the natural teeth adjacent to the 
implant is higher.

\section{Conclusions}

Generally, the contact between two teeth is simplified by a point contact, and the floss can enter the gap between two teeth under pressure [13]. The interproximal space between two teeth will be closed by medical technique to a point contact [14]. What's more, this boundary is dynamic and varies with age, teeth alignment, crowding, masticatory force [9]. The contact area can be evaluated by the strength, the location, the shape and the size of contact. However, research about the contact between dental implants and natural adjacent teeth is rare, which is also ignored or simplified in the finite element analysis.

According to the results of finite element analysis, it can be seen that stress distribution of five groups is almost the same whatever the first molar implant contact with its adjacent teeth, and the stress concentration of the alveolar bone is obvious when there is no contact between two adjacent teeth. Compared with the group $\mathrm{C} 0$, the stress of alveolar bone surrounding the implant in other groups decreased $88.08 \%$ at most, but the stress of cancellous bone surrounding the natural teeth increased $59.42 \%$. The load implant suffered can be divided in lateral load and vertical load. The lateral load can be distributed by "pushing" adjacent teeth to natural teeth, and vertical load can be distributed by friction adjacent teeth to the natural teeth, which reduces the stress of implant and alveolar bone around the implant when implant has a contact area with adjacent tooth. With the area of contact becoming bigger, the lateral load and vertical load adjacent teeth suffered is increasing, and the stress of alveolar bone and periodontal ligament around the natural teeth is increasing, but the stress of alveolar bone around the implant is decreasing.

Without the function of distribution of the periodontal ligament, the implant is more unbearable the lateral load compared with the original natural tooth. Therefore, small area contact is beneficial to disperse the lateral load applied in the implant and reduce the stress of the bone interface. At the same time, the stress in the periodontal ligament and alveolar bone around natural teeth adjacent to the implant is not too high. During the process of modeling, there are two rough designs can be improved in further research: (1) The influence of lateral load applied in the natural adjacent teeth is not considered; (2) The physiological loads applied in the finite element models are simplified to static load, which are generally dynamic load in the oral cavities.

Some conclusions can be draw from the finite element analysis: (1) The stress of the bone interface and implant was significantly decreased when implant was in contact with the natural adjacent teeth. (2) It is effectively to distribute the load implant suffered, especially to reduce the stress generated by the lateral load when the first molar implant has a small area contact with its adjacent teeth. It could avoid an excessive stress in the periodontal ligament and alveolar bone around the natural teeth. (The proper contact area with the second premolar is $1.772 \mathrm{~mm}^{2}-5.295 \mathrm{~mm}^{2}$, and the contact area with the second molar is $2.095 \mathrm{~mm}^{2}-6.58 \mathrm{~mm}^{2}$ ).

\section{References}

[1] Lv J., Mi C. Research progress on biomechanics of periodontal ligament. International Journal of Stomatology, Vol. 41, Issue 3, 2014, p. 362-364.

[2] Toms S. R., Lemons J. E., Bartolucci A. A. Nonlinear stress-strain behavior of periodontal ligament under orthodontic loading. American Journal of Orthodontics and Dentofacial Orthopedics, Vol. 122, Issue 2, 2002, p. 174-179.

[3] Li T. Optimum selection of the dental implant diameter and length in the posterior mandible with poor bone quality - A 3D finite element analysis. Applied Mathematical Modelling, Vol. 35, Issue 1, 2011, p. $446-456$.

[4] De Santis R. Reverse engineering of mandible and prosthetic framework: Effect of titanium implants in conjunction with titanium milled full arch bridge prostheses on the biomechanics of the mandible. Journal of Biomechanics, Vol. 47, Issue 16, 2014, p. 3825-3829. 
[5] Peixoto H. E. Rehabilitation of the atrophic mandible with short implants in different positions: A finite elements study. Materials Science and Engineering: C, Vol. 80, 2017, p. 122-128.

[6] Zhao Y. The remodeling of alveolar bone supporting the mandibular first molar with different levels of periodontal attachment. Medical and Biological Engineering and Computing, Vol. 51, Issue 9, 2013 , p. 991-997.

[7] Cornacchia T. P. M. 3D finite element analysis on esthetic indirect dental restorations under thermal and mechanical loading. Medical and Biological Engineering and Computing, Vol. 48, Issue 11, 2010, p. 1107-1113.

[8] Ding X. Effect of orthotropic material on finite element modeling of completely dentate mandible. Materials and Design, Vol. 84, 2015, p. 144-153.

[9] Sarig R. The arrangement of the interproximal interfaces in the human permanent dentition. Clinical Oral Investigations, Vol. 17, Issue 3, 2013, p. 731-738.

[10] Liu Z., Fan Y., Qian Y. Comparative evaluation on three-dimensional finite element models of the temporomandibular joint. Clinical Biomechanics, Vol. 23, 2008, p. 53-58.

[11] Roy S., Basu B. Mechanical and tribological characterization of human tooth. Materials Characterization, Vol. 59, Issue 6, 2008, p. 747-756.

[12] Testa M., Di Marco A., Pertusio R., Van Roy P., Cattrysse E., Roatta S. A validation study of a new instrument for low cost bite force measurement. Journal of Electromyography and Kinesiology, Vol. 30, 2016, p. 243-248.

[13] Huang M., Wang M. Relationship between the interproximal interface of adjacent teeth and food impaction. International Journal of Stomatology, Vol. 3, 2016, p. 303-308.

[14] Zhang J. Clinical treatment and therapeutic effect of food contact ring forming contact bonding technology. Journal of Sichuan University (Medical Science Edition), Vol. 44, Issues 5, 2013, p. $845-857$. 